\title{
Facile Synthesis of NiSe:Zn Nanoparticles by Method Variation and their Morphological Studies
}

\author{
M. SASTHAA BEGUM and A. JAFAR AHAMED \\ Department of Chemistry, Jamal Mohamed College, Tiruchirappalli, Tamilnadu, India \\ msasthaa@gmail.com
}

Received 25 December 2015 / Accepted 11 January 2016

\begin{abstract}
The nano crystals were synthesized by two different routes such as thermal reduction process and coprecipitation process and the difference in morphology of NiSe: $\mathrm{Zn}$ was studied. $\mathrm{Zn}$ doped nickel selenide was synthesized from the reaction of nickel chloride $\left(\mathrm{NiCl}_{2} \cdot 6 \mathrm{H}_{2} \mathrm{O}\right)$, sodium selenite $\left(\mathrm{Na}_{2} \mathrm{SeO}_{3}\right)$ and anhydrous zinc chloride $\left(\mathrm{ZnCl}_{2}\right)$ using poly- $N$-Vinyl-2pyrrolidone (PVP) as capping agent and hydrazine hydrate $\left(\mathrm{N}_{2} \mathrm{H}_{4} \cdot \mathrm{H}_{2} \mathrm{O}\right)$ as reductant in thermal reduction process (method-1). In coprecipitation process it was prepared by the reaction of $\mathrm{NiCl}_{2} \cdot 6 \mathrm{H}_{2} \mathrm{O}, \mathrm{Na}_{2} \mathrm{SeO}_{3}$ and anhydrous $\mathrm{ZnCl}_{2}$ using certain amount of PVP which acts as reductant as well as capping agent (method-2). The products were characterized by $\mathrm{x}$-ray diffraction (XRD), Field Emission Scanning electron microscopy (FE-SEM), Energy dispersive $x$-ray spectroscopy (EDX), Fourier Transform Infra red spectroscopy (FT-IR), Visible Ultra violet spectroscopy (UV) and Photoluminescence (PL) techniques.
\end{abstract}

Keywords: Nanocrystals, Synthesis, Morphological studies, Facile synthesis

\section{Introduction}

Recently there is extensive attention given to the preparation and characterization of metal chalcogenides due to their properties and potential applications. There are different methods of synthesis used for preparing metal chalcogenides like chemical method, hydrothermal process, solvothermal, CVD, ultrasonic synthesis ${ }^{1-5}$, using various surfactants. The doped metal chalcogenides have attracted more attention and many such chalcogenides are successfully synthesized ${ }^{6-15}$. In this paper an attempt to synthesise $\mathrm{Zn}$ doped NiSe using two different modes - one using the reducing agent hydrazine hydrate at $100{ }^{\circ} \mathrm{C}$ with PVP as capping agent and other using only PVP at room temperature. An attempt is also taken to study the effect of varying the modes of synthesis on the size, morphology, optical and fluorescence properties.

\section{Experimental}

All the chemicals used during the experiments were analytical grade and were used as such without further purification. 


\section{Synthesis of zinc doped NiSe - Thermal reduction using PVP (Method-1)}

$\mathrm{NiCl}_{2} \cdot 6 \mathrm{H}_{2} \mathrm{O}(0.2 \mathrm{M})$ and $\mathrm{Na}_{2} \mathrm{SeO}_{3}(0.2 \mathrm{M})$, with a stoichiometric ratio of $1: 1$ were dissolved in $50 \mathrm{~mL}$ of deionised water. To this solution certain amount of PVP $(0.0002 \mathrm{M})$ was added with constant stirring. $20 \mathrm{~mL}$ of hydrazine hydrate and $25 \mathrm{~mL}$ of anhydrous $\mathrm{ZnCl}_{2 \mathrm{~s}}(0.02 \mathrm{M})$ were added to the above solution and kept for heating at $100{ }^{\circ} \mathrm{C}$ for $5 \mathrm{~h}$. On adding hydrazine hydrate, greenish yellow colour changed to black. The black precipitate obtained was washed with deionised water and acetone several times, to remove PVP and impurities. Finally it was dried at $100{ }^{\circ} \mathrm{C}$ for 2 hours.

\section{Synthesis of zinc doped NiSe - Coprecipitation route using PVP (Method-2)}

$\mathrm{NiCl}_{2} \cdot 6 \mathrm{H}_{2} \mathrm{O}(0.2 \mathrm{M})$ and $\mathrm{Na}_{2} \mathrm{SeO}_{3}(0.2 \mathrm{M})$ with a stoichiometric ratio of 1:1 were dissolved in $25 \mathrm{~mL}$ of deionised water. The addition was carried out drop by drop with constant stirring. $10 \mathrm{~mL}$ of anhydrous $\mathrm{ZnCl}_{2}(0.02 \mathrm{M})$ and certain amount of PVP $(0.0002 \mathrm{M})$ were added to the above solution. This solution was kept in a magnetic stirrer at room temperature for 4 hours. The yellowish green precipitate obtained was washed with deionised water and acetone several times to remove PVP and other impurities. Finally it was dried at $100{ }^{\circ} \mathrm{C}$ for 1.30 hours.

\section{Results and Discussion}

Figure 1 and 2 show XRD patterns of Zn doped NiSe prepared on the basis of method-1 and 2 . The XRD analysis showed a considerable difference in the morphology of $\mathrm{Zn}$ doped NiSe. The one obtained by method- 1 has lattice contants $a=b=0.806 \mathrm{~nm}, c=2.215 \mathrm{~nm}, \alpha=\beta=\gamma \neq 90^{\circ}$ indicating rhombohedral structure and the one obtained by method-2 has lattice constants $a=1.214 \mathrm{~nm}, b=0.575 \mathrm{~nm}, c=1.539 \mathrm{~nm}, \alpha=\gamma=90^{\circ}, \beta=114.13^{\circ}$ indicating monoclinic structure (calculated using JCPDS). Since the precursors, their concentration and capping agent taken were same in both the methods, the difference in the phase of zinc doped nickel selenides could be attributed to the difference in temperature and presence of hydrazine hydrate ${ }^{17}$ in method-1 and absence of the same in method-2.

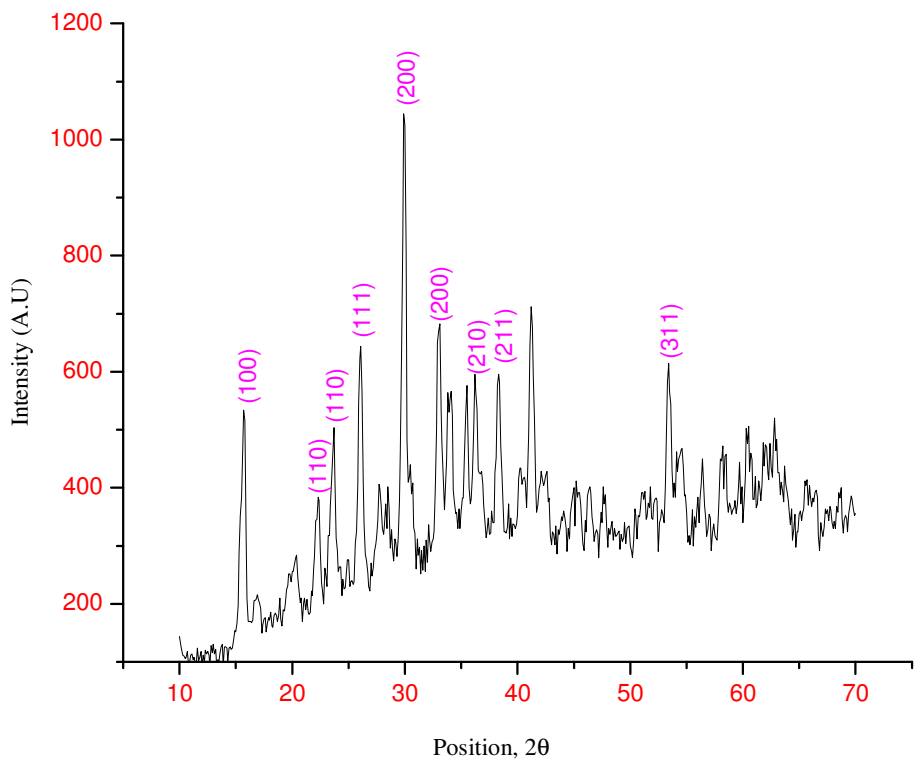

Figure 1. XRD patterns of NiSe:Zn by method-1 


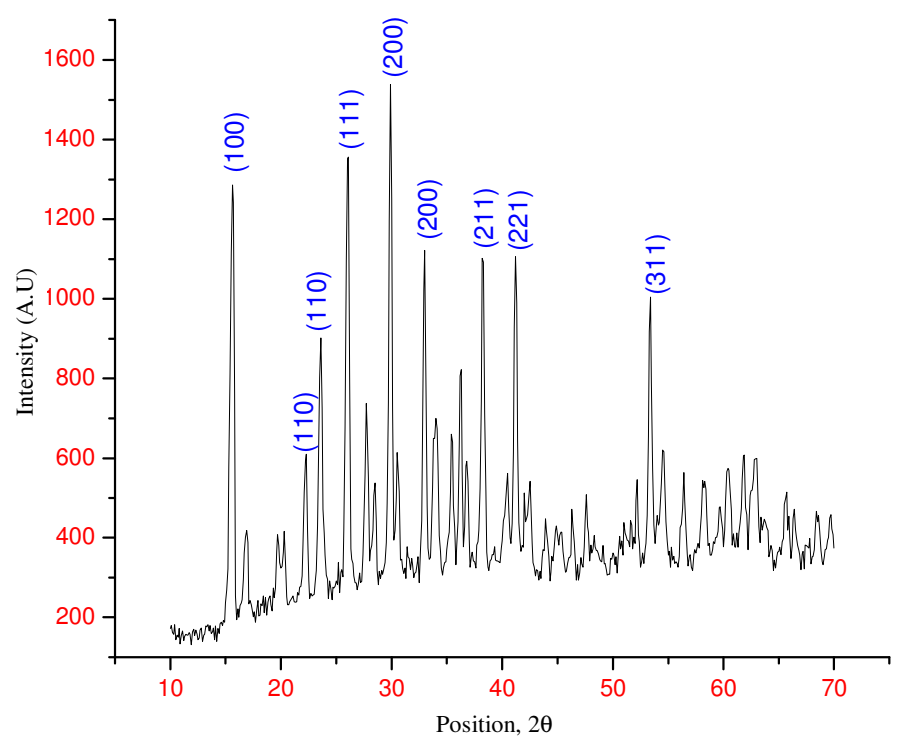

Figure 2. XRD patterns of NiSe:Zn by method-2

The sharp peaks obtained in both the methods indicated crystallinity of the samples. The intensity of the XRD plot obtained by method-2 was found to be higher than the one obtained by method-1. This indicated clearly the electron density inside the unit cell obtained by method- 2 was greater than the one obtained by method- 1 . The average crystallite size was calculated using Scherrer's formula. It was found to be $16.57 \mathrm{~nm}$ by method- 1 and $22.08 \mathrm{~nm}$ by method- 2 . Hence, it can be concluded that there was some difference in the crystallite size by method variation. Effect of doping on the strain of the crystal was calculated using the expression: $\epsilon=\beta \cos \theta / 4$. It was found to be $20.95 \times 10^{-4}$ in the case of method- 1 and $15.93 \times 10^{-4}$ in the case of method- 2 . It was found to be more in the first case than the second. The change in phase of zinc doped nickel selenide by method variation could also be attributed to the strain caused in the crystal.

Dislocation density was calculated using the expression: $\partial=15 \beta \cos \theta / 4$ a D. It was found to be $2.352 \times 10^{15}$ by method -1 and $0.881 \times 10^{15}$ by method- 2 . The decrease in dislocation density in the case of second method showed increased crystallinity, which was also in agreement with the XRD plots obtained.

Figure $3 \& 4$ shows the FE-SEM analysis of $\mathrm{Zn}$ doped NiSe obtained by method- 1 and 2 . There was observable difference seen in the morphology of the crystals obtained by method variation. It was found to have honey comb like structure by thermal reduction process and cubic box like structure by coprecipitation process. The distribution of particles was found to be similar and homogeneous in both the cases. The average particle size calculated for the nano particles obtained by method-1 was found to be $29.74 \mathrm{~nm}$ while it was obtained in micro dimension by method-2. The average particle size was found to be $2.734 \mu \mathrm{m}$.

EDX result of $\mathrm{Zn}^{2+}$ doped NiSe obtained for method- 1 and 2 is shown in Figure $5 \& 6$. The EDX result confirmed the presence of $\mathrm{Ni}$, Se and $\mathrm{Zn}$ in the sample. Figure $7 \& 8$ show UV-Visible spectra obtained by method-1 and 2 . Band gap energy was calculated using, $\mathrm{Eg}=\mathrm{hc} / \lambda$. There was observable difference noticed in band gap energy of zinc doped nickel 
selenides obtained by method variation. Eg value was found to be $3.54 \mathrm{eV}$ for the crystal obtained by method- 1 while it was found to be $1.24 \mathrm{eV}$ for the crystals obtained by method- 2 . This showed that band gap energy decreases to greater extent when the mode of synthesis was coprecipitation in comparison to thermal reduction process. This in turn increases the conductivity value of nickel selenide.

Molar absorptivity was calculated using the expression: $\epsilon=\mathrm{A} / \mathrm{cl}$. It was found to be very high for the nano crystals obtained by method-2 than the one obtained by method- 1 . The molar absorptivity values can be given as, method-1: $877.5 \times 10^{-7} \mathrm{~cm}$ and method-2: $2147.5 \times 10^{-7} \mathrm{~cm}$. This showed that light absorbing functions have increased to a greater extent for the doped nickel selenide obtained by method- 2 compared to method-1. Further there was large bathochromic shift seen in the case of zinc doped NiSe obtained by coprecipitation than the one obtained by thermal reduction.
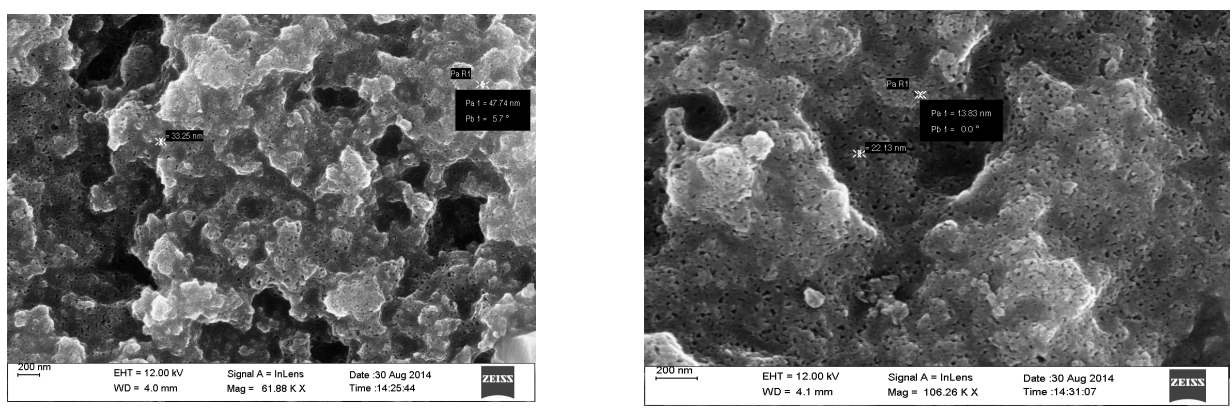

Figure 3. SEM images of NiSe:Zn obtained by method - 1
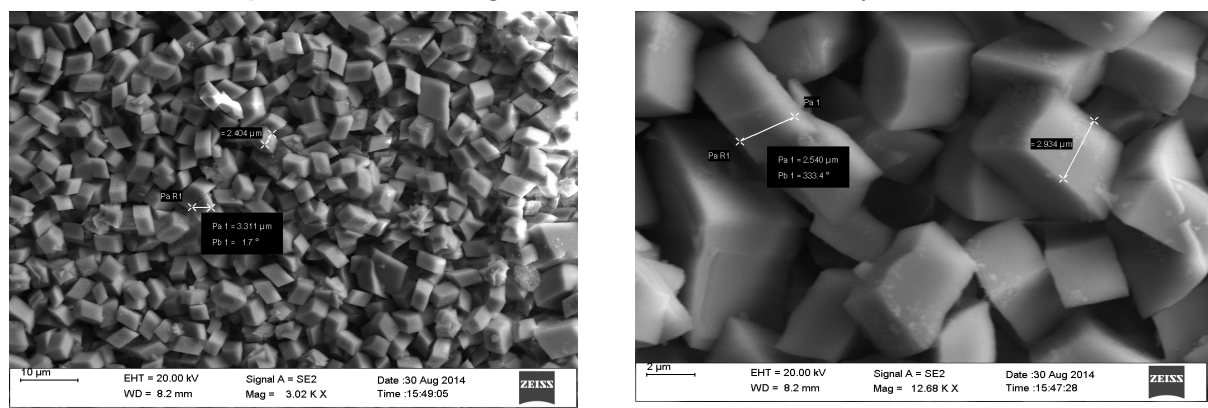

Figure 4. SEM images of NiSe:Zn obtained by method - 2

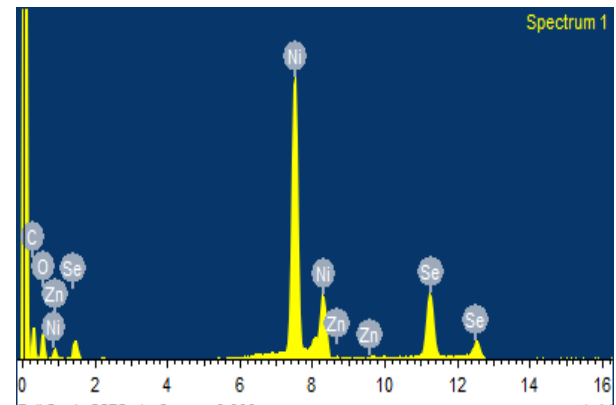

Full Scale 2575 cts Cursor: 0.000

Figure 5. EDX results obtained for NiSe:Zn by method-1

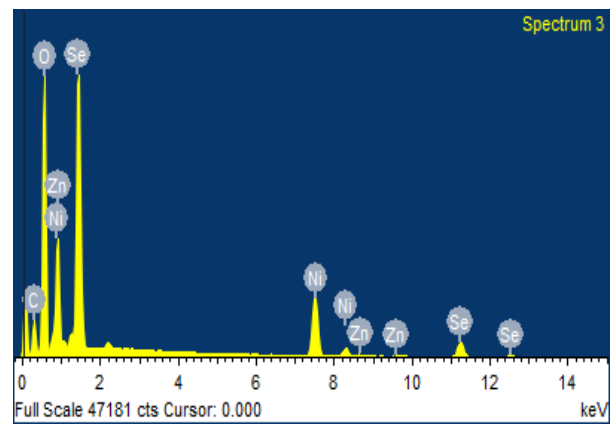

Figure 6. EDX results obtained for NiSe:Zn by method -2 


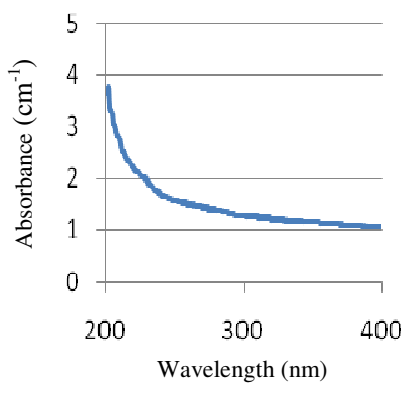

Figure 7. UV-Visible spectra of NiSe:Zn obtained by method-1

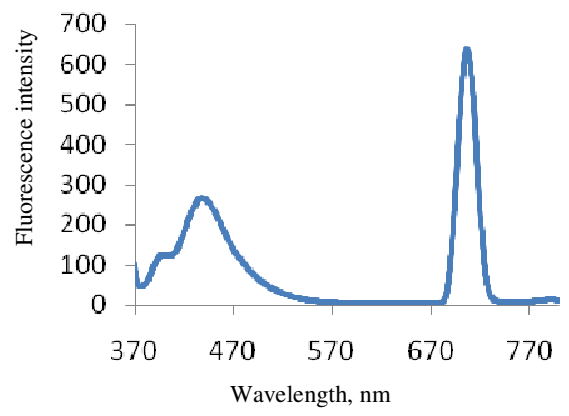

Figure 9. PL spectra of NiSe:Zn obtained by method-1

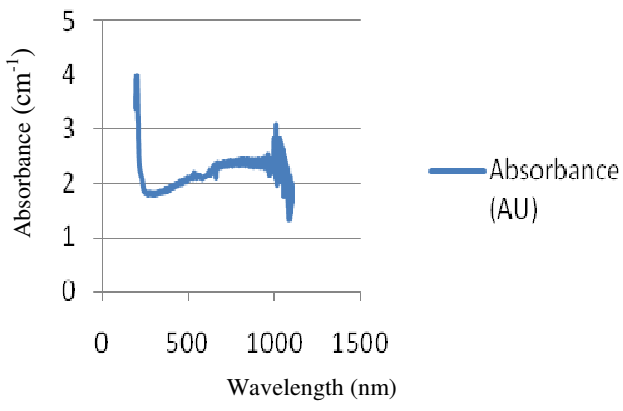

Figure 8. UV-Visible spectra of NiSe:Zn obtained by method-2

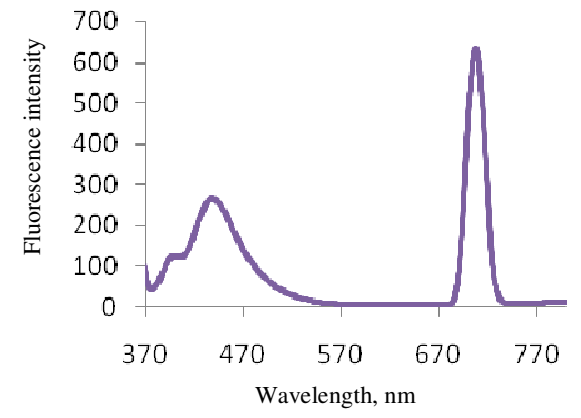

Figure 10. PL spectra of NiSe:Zn obtained by method-2

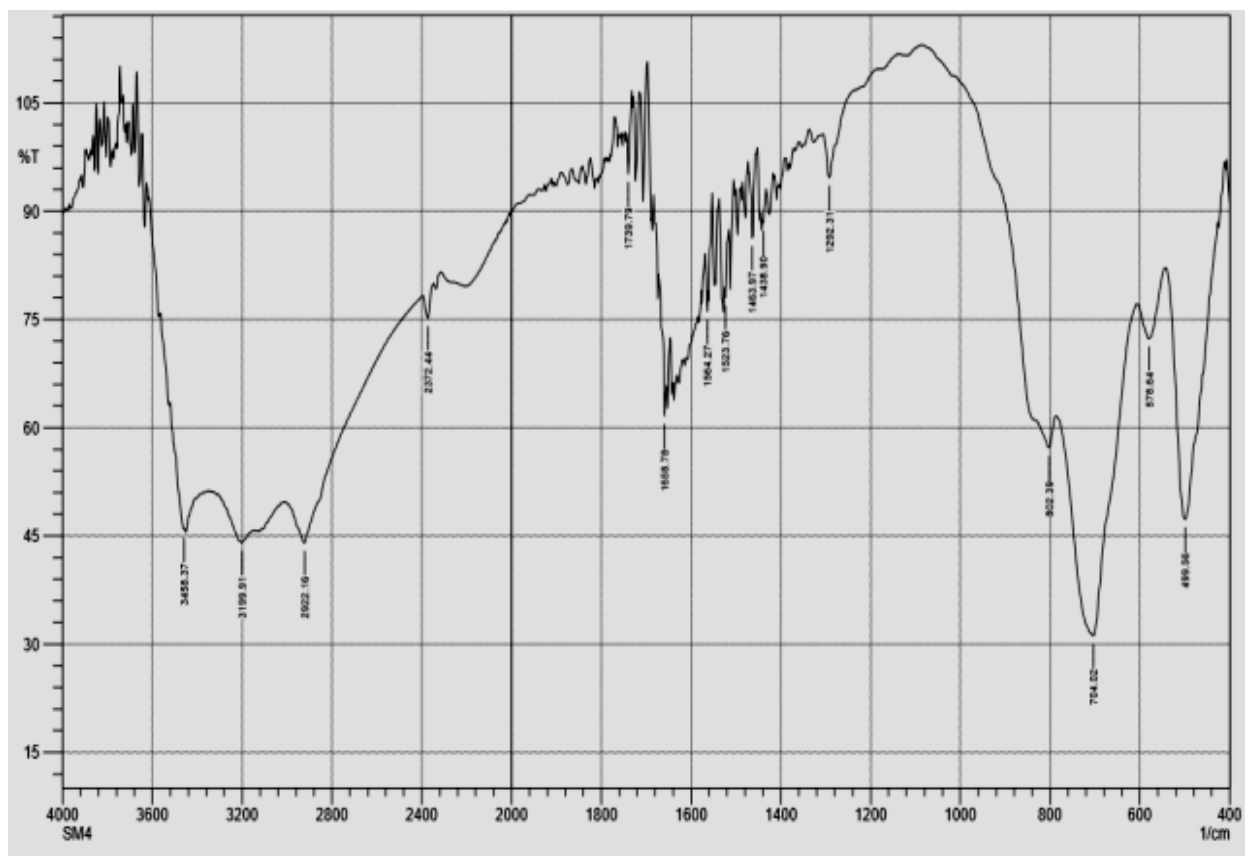

Figure 11. FT-IR spectra of NiSe:Zn obtained by method-1 


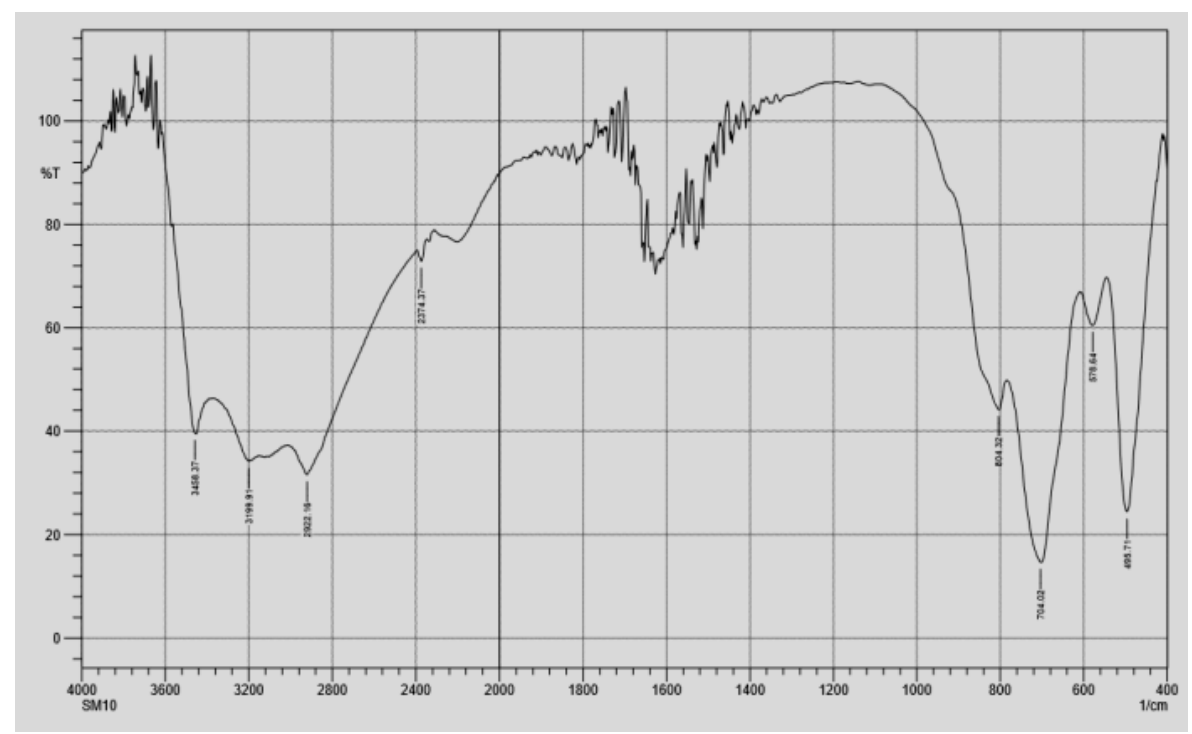

Figure 12. FT-IR spectra of NiSe:Zn obtained by method-2

Figure $9 \& 10$ shows the PL spectra of zinc doped NiSe obtained by method-1 and method-2. The PL spectra obtained by method variation of zinc doped NiSe was comparable. This showed that the photoluminescence property does not vary by changing the modes of synthesis of nano particles. There was red emission observed in the wavelength range of 690-700 nm. In both cases of zinc doped nickel selenides, there were two peaks observed corresponding to blue and red emission. This showed that $\mathrm{Zn}^{2+}$ ion were incorporated into the nickel selenide crystal lattice. The high intensity peaks indicates the increased quantum efficiency in both cases. Figure 11 and 12 show the FT-IR spectra of NiSe:Zn obtained by method- 1 and 2 .

IR spectra of both Zn doped NiSe crystals obtained by method- 1 and 2 were comparable showing the presence of $\mathrm{Ni}$-Se bond. As oxides are the common impurities which depend on the chemical composition of chalcogenides, some band related to vibrations of oxygen atoms bonded to basic elements and carbon impurity atoms were noticed in the spectra. Vibrations related to Ni-O bond, $\mathrm{Zn}-\mathrm{O}$ and $\mathrm{C}=\mathrm{O}$ bond were observed. The concentration of adsorbed $\mathrm{CO}_{2}$ molecule was found to be more in case of nano crystals obtained by method-1, which was seen by the extra bands observed in between $1658-1438 \mathrm{~cm}^{-1}$.

\section{Conclusion}

- $\mathrm{Zn}^{2+}$ doped nickel selenides were synthesized successfully by thermal reduction and coprecipitation process.

- XRD analysis revealed the presence of rhombohedral phase for the one obtained by method-1 and monoclinic phase by method-2. The average crystallite size of NiSe:Zn was found to be $16.57 \mathrm{~nm}$ and $22.08 \mathrm{~nm}$ by method- 1 and 2 respectively. There was some difference noticed in strain of the crystals and observable difference noticed in dislocation density.

- FE-SEM showed the presence of honey comb like nano crystals for zinc doped NiSe obtained by method- 1 while cubic box like structure for the one obtained by method- 2 . There was much difference noticed in particle size. NiSe:Zn obtained by method-1 had 
nano dimension while the one obtained by method- 2 had micro dimension. The EDX analysis confirmed the presence of $\mathrm{Ni}, \mathrm{Se}$ and $\mathrm{Zn}$ in both the samples.

- PL spectra of zinc doped NiSe were almost comparable for both the crystals.

- There was some difference noticed in the band gap energy calculated from UV-Visible spectra of both the samples.

- There was observable difference noticed in molar absorptivity of zinc doped NiSe obtained by method variation. This showed that light absorbing function changes with the change in the mode of synthesis.

- $\quad$ FT-IR spectra of the samples obtained by the method variation were comparable and confirmed the various vibration modes of the bonds.

\section{References}

1. Hankare P P, Jadhav B V, Garadkar K M, Chate P A, Mulla I S and Delekar S O, J Alloys Compounds, 2010, 490(1-2), 228-231; DOI:10.1016/j.jallcom.2009.09.132

2. Azam Sobhani, Fatemeh Davar and Masoud Salavati-Niasari, Appl Surface Sci., 2011, 257(18), 7982-7987; DOI:10.1016/j.apsusc.2011.04.049

3. Masoud Salavati Niasari, Azam Sobhani and Fatemeh Davar, J.Alloys Compounds, 2010, 507(1), 77-83; DOI:10.1016/j.jallcom.2010.06.062

4. Azam Sobhani and Masoud Salavati-Niasari, Superlattices Microstructures, 2014, 65, 79-90; DOI:10.1016/j.spmi.2013.10.030

5. Azam Sobhani, Masoud Salavati-Niasari and Fatemeh Davar, Polyhedron, 2012, 31(1), 210-216; DOI:10.1016/j.poly.2011.09.017

6. Rahdar A, Asnaasahri Eivari H and Sarhaddi R, Indian J Sci Tech., 2012, 5(1), 1855-1858.

7. Kole A K and Kumbhakar P, Appl Nanosci., 2012, 2(1), 15-23; DOI:10.1007/s13204011-0036-X

8. Soundararajan D, Yoon J K, Kim Y I, Kwon J S, Park C W, Kim S H and Ko J M, Int J Electrochem Sci., 2009, 4, 1628-1637.

9. Sunil Kumar, Nitu Kumari, Sanjeev Kumar, Sanyog Jain and Verma N K, Appl Nanosci., 2012, 2, 437-443; DOI:10.1007/s13204-011-0056-6

10. Murugadoss G and Rajesh Kumar M, Appl Nanosci., 2014, 4(1), 67-75; DOI:10.1007/s13204-012-0167-8

11. Kanupriya Sharma and Ashok Kumar, AIJRSTEM., 2014, 8(1), 75-79.

12. Khalid T Al- Rasoul, Nada K Abbas and Zainb J Shanan, Int J Electrochem Sci., 2013, 8, 5594-5604.

13. Khairy M and Zakaria W, Egyptian J Petroleum., 2014, 23(4), 419-426; DOI:10.1016/j.ejpe.2014.09.010

14. Sandeep Gohar and Pathak R K, Oriental J Chem., 2013, 29(4), 1469-1474; DOI: 10.13005/ojc/290424.

15. Yao-Ming Hao, Shi-Yun Lou, Shao-Min Zhou, Rui-Jian Yuan, Gong-Yu Zhu and Ning Li, Nanoscale Res Lett., 2012, 7, 100; DOI:10.1186/1556-276X-7-100

16. Cristina E Hoppe, Massimo Lazzari, Ivan Pardin As-Blanco and M Arturo LopezQuintela, Langmuir, 2006, 22(16), 7027-7034; DOI:10.1021/la060885d

17. Monica Debbarma, Soma Das and Mitali Saha, Adv Manuf., 2013, 1(2), 183-186. 